\title{
Aneuploidy among androgenic progeny of hexaploid triticale (XTriticosecale Wittmack)
}

\author{
Sylwia Oleszczuk · Julita Rabiza-Swider • \\ Janusz Zimny • Adam J. Lukaszewski
}

Received: 27 September 2010/Revised: 2 December 2010/Accepted: 3 December 2010/Published online: 19 December 2010

(C) The Author(s) 2010. This article is published with open access at Springerlink.com

\begin{abstract}
Doubled haploids are an established tool in plant breeding and research. Of several methods for their production, androgenesis is technically simple and can efficiently produce substantial numbers of lines. It is well suited to such crops as hexaploid triticale. Owing to meiotic irregularities of triticale hybrids, aneuploidy may affect the efficiency of androgenesis more severely than in meiotically stable crops. This study addresses the issue of aneuploidy among androgenic regenerants of triticale. Plant morphology, seed set and seed quality were better predictors of aneuploidy, as determined cytologically, than flow cytometry. Most aneuploids were hypoploids and these included nullisomics, telosomics, and translocation lines; among 42 chromosome plants were nulli-tetrasomics. Rye chromosomes involved in aneuploidy greatly outnumbered wheat chromosomes; in $\mathrm{C}_{0}$ rye chromosomes $2 \mathrm{R}$ and $5 \mathrm{R}$ were most frequently involved. While the frequency of nullisomy $2 \mathrm{R}$ was fairly constant in most cross combinations, nullisomy $5 \mathrm{R}$ was more frequent in the most recalcitrant combination, and its frequency increased with time spent in culture with up to $70 \%$ of green plants recovered late being nullisomic $5 \mathrm{R}$. Given that $5 \mathrm{R}$ was not
\end{abstract}

Communicated by M. Jordan.

S. Oleszczuk · J. Zimny

Plant Breeding and Acclimatization Institute, National Research

Institute, Radzikow 05-870 Blonie, Poland

J. Rabiza-Swider

Department of Ornamental Plants, Warsaw University of Life

Sciences, Nowoursynowska 166, 02-787 Warsaw, Poland

A. J. Lukaszewski ( $\square)$

Department of Botany and Plant Sciences,

University of California, Riverside, CA 92521, USA

e-mail: adam.lukaszewski@ucr.edu involved in meiotic aberrations with an above-average frequency, it is possible that its absence promotes androgenesis or green plant regeneration. Overall, aneuploidy among tested combinations reduced the average efficiency of double haploid production by $35 \%$ and by $69 \%$ in one recalcitrant combination, seriously reducing the yield of useful lines.

Keywords Aneuploid · Doubled haploid · Androgenesis . Triticale
Abbreviations
DH Doubled haploid
MI Meiotic metaphase I
PMC Pollen mother cell
QTL Quantitative trait loci

\section{Introduction}

Doubled haploid techniques have established themselves as useful tools in plant breeding and genetic research. As of 2005 doubled haploids (DHs) had been produced in over 230 different crops (Forster and Thomas 2005). DH lines can be produced in several ways, by distant hybridization (this includes chromosome elimination in early embryo development), by androgenesis or by gynogenesis (Forster et al. 2007). Each method has some advantages and disadvantages and not all are applicable or efficient in all crops. As discussed by Forster and Thomas 2005 the advantage of DH in breeding is in (perceived) time savings between the initial cross and large-scale testing of developed lines. Doubled haploids quickly provide large 
amounts of seed for replicated field trials. The advantage of DHs in genetic research is that perfectly homozygous lines can be produced relatively quickly and permit large scale replicated trials for detection of quantitative trait loci (QTLs) and their allocation to specific, sometimes very small, chromosome regions. In all cases, the critical issue in the use of double haploids is the efficiency of their production. This efficiency can be measured in several ways but it can always be brought down to the ultimate factor: how many useful lines can be produced in a given time with available labor and resources. For those involved in the DH development, the critical measure of efficiency is the number of green plants (or DH lines) produced. However, not all green plants need to be useful in breeding or research. Given that stability/uniformity is the main advantage of the DH lines, any deviation from it, including that caused by aneuploidy, is detrimental and in most cases will disqualify a line from any research or breeding.

Triticale is a new cereal that in ca. 135 years since the first man-made wheat-rye hybrids were created (Lelley 2006; Lukaszewski and Gustafson 1987; Struss and Röbbelen 1989) has established itself as a competitive crop in certain environments. Unlike its progenitors, wheat and rye, triticale is generally amenable to anther culture and in most instances produces reasonable yields of haploids. First commercial cultivars of triticale developed with the aid of androgenesis have been released (Z. Banaszak, pers. comm.) and the method is routinely used in breeding with thousands of DHs produced every year $(\mathrm{H}$. Wos, N. Darvey, Z. Banaszak, pers. comm.). The androgenic response in triticale appears to be under genetic control but in most cases, sufficiently large populations can be generated. However, despite a considerable progress in (indirect) breeding for meiotic stability, triticale still has chromosome pairing issues and tends to show a fair proportion of aneuploids among sexually derived progenies, more so in hybrids than established lines (Lelley 2006; Lukaszewski and Gustafson 1987). Perhaps for this reason, the issue of efficiency of the $\mathrm{DH}$ production and the quality of the recovered $\mathrm{DH}$ lines requires more attention in triticale than in most better established and more meiotically stable crops, such as wheat or barley.

The wider context of this study was to generate sufficiently sized mapping populations of the DH triticale lines. Aneuploidy severely complicated this effort and appeared as an issue deserving to be analyzed with an additional effort. However, since from the start the emphasis was on the mapping populations and not the process of androgenesis itself, some data on population sizes and frequencies of specific events were never collected. Still, it appears that the issue of aneuploidy in triticale $\mathrm{DH}$ lines is important enough to be addressed.

\section{Materials and methods}

\section{Plant material}

Triticale haploids and DHs were generated by androgenesis from the $F_{1}$ hybrids involving the following winter triticales: cv. Presto, originally obtained from the late Dr. E. Tymieniecka, then Poznan Plant Breeding, Poland; Presto RM1B, Presto RM2 and Presto FC2 produced by Lukaszewski (2006); cv. Mungis obtained from Dr. B. Schinkel, Lochow-Petkus GmbH, Germany; cv. Krakowiak from Dr. H. Wos, HR Strzelce, Poland; cv. Stan 1 from Dr. S. Nalepa, then Resource Seed Inc., Gilroy CA, USA; breeding line NE422T from Dr. P. S. Baenziger, Univ. of Nebraska, Lincoln NE, USA. The following $F_{1}$ combinations were processed: Presto $\times$ Mungis, Presto $\times$ Krakowiak, Presto $\times$ NE422T, Mungis $\times$ Krakowiak, Mungis $\times$ Stan 1 , and NE422T $\times$ Stan 1 . In combinations involving cv. Presto some hybrids were of the original cultivar while others were of Presto with engineered chromosome $1 \mathrm{R}$, usually in equal proportions of the two (standard vs. engineered 1R) in each varietal combination.

\section{Anther culture and plant regeneration}

The androgenesis protocol was essentially the same as that used for the chromosome number reduction project in Lolium $\times$ Festuca hybrids (Kopecky et al. 2005). Triticale tillers with anthers presumed to be in the late microspore stage were cut from plants grown in the greenhouse, checked for the microspore development stage in anthers live-stained with $1 \%$ acetocarmine, and refrigerated for 2-5 days at $2-4^{\circ} \mathrm{C}$ in the N6 liquid medium supplemented with $1 \mathrm{mg}$ 2.4D per 1L (Ryöppy 1997).

The regenerated green plants were transplanted from test tubes to soil, grown in flats in the greenhouse for 3-4 weeks and with one exception (Presto $\times$ Mungis) colchicine treated. This treatment was by submersion of the tillering crowns of plants in an aerated aqueous $0.1 \%$ solution of colchicine with $3 \%$ DMSO for ca. $7.5 \mathrm{~h}$. Treated plants were rinsed in cold running water for ca. $15-20 \mathrm{~h}$, refrigerated at $2-4^{\circ} \mathrm{C}$ for $2-5$ days, planted into flats in a soil mix and placed in a cold room at ca. $5-7^{\circ} \mathrm{C}$ at $12 \mathrm{~h}$ day/12 h night for $7-8$ weeks. After the vernalization requirement was complete, the treated plants were transplanted into $18 \mathrm{~cm}$ pots and grown to maturity. All emerging heads were bagged to eliminate all possibility of cross pollination and were hand-threshed at maturity. In the Presto $\times$ Mungis combination, all progeny obtained was from spontaneous chromosome doubling events. 
Flow cytometry

The measurements of the DNA contents were done on a population of spontaneously DHs of the Presto $\times$ Mungis combination. Ploidy levels of the regenerated plants were evaluated by fluorescence (DAPI) using a Partec II (Germany) flow cytometer (FC). Samples were prepared according to Galbraith et al. (1983) with some modifications. Leaf fragments were chopped with a sharp razor blade in a Petri dish with $2 \mathrm{ml}$ of the nuclei-isolation buffer (0.1 M Tris, $2.5 \mathrm{mM} \mathrm{MgCl}_{2} \cdot 6 \mathrm{H}_{2} \mathrm{O}, 85 \mathrm{mM} \mathrm{NaCl}, 0.1 \%$ (v/v) Triton X-100) containing DAPI and the cell suspension was filtered through a $30-\mu \mathrm{m}$ filter. The ploidy levels of regenerants were determined by comparing the $\mathrm{G} 1$ peaks of the analyzed samples to internal controls, here both parents, cv. Presto and Mungis. Plants with the peaks of the DNA contents distribution clearly separated from the controls were considered aneuploid.

SDS-polyacrylamide gel electrophoresis

All DH lines from hybrids including engineered chromosomes 1R (Lukaszewski 2006) were screened for the presence/absence of these chromosomes in a two-step SDS-polyacrylamide gel electrophoresis (SDS-PAGE) as described by Brzezinski and Lukaszewski (1998) and Lukaszewski (2006). Presence/absence or changes in protein band intensities were considered indicative of the status of group-1 chromosomes.

Cytology

Root tips for karyotyping were collected either from seed germinated on wet filter paper in Petri dishes, or from plants grown in a hydroponic culture in an aerated full strength GroResearch Gromagnon 9-5-18 All-Purpose-Nutrient solution from American Hydroponics, Arcata, CA USA. For collections from germinating seedlings, seed were placed on wet filter paper in Petri dishes for 2-3 days at room temperature, refrigerated at ca. $2^{\circ} \mathrm{C}$ for $2-3$ days, transferred to room temperature $\left(23-24^{\circ} \mathrm{C}\right)$ for $24 \mathrm{~h}$ and collected to ice water for ca. $27 \mathrm{~h}$. Depending on the intended cytological protocol, roots were either fixed in $45 \%$ acetic acid overnight and squashed, or fixed in a Carnoy solution (3:1 mixture of absolute ethanol:glacial acetic acid). Roots from the hydroponic-grown plants were collected to ice water for ca. $27 \mathrm{~h}$ and fixed in the Carnoy solution. For the analyses of meiotic metaphase I (MI) chromosome pairing one anther from a spikelet of an ear was excised and live-squashed in a drop of $1 \%$ acetocarmine. If MI was present, the remaining two anthers were fixed in the Carnoy solution.

All material in the Carnoy solution was fixed for 7 days at $37^{\circ} \mathrm{C}$, stained in $1 \%$ acetocarmine for $2 \mathrm{~h}$, re-fixed and stored at $-20^{\circ} \mathrm{C}$ until used. All preparations of this type of material were made by squashing in a drop of $45 \%$ acetic acid using the two-coverslip technique. All C-banding on the $45 \%$ acetic acid- fixed root tips was according to Lukaszewski and $\mathrm{Xu}$ (1995); all C-bandning on the Carnoy-fixed material, whether root tips or anthers, was according to Giraldez et al. (1979).

In situ probing with labeled DNA was done according to the protocol of Dr. T. Endo, Kyoto University, Japan (Masoudi-Nejad et al. 2002). Total genomic DNA of rye was labeled with digoxigenin and detected by the antidigoxigenin-fluorescein using standard kits and protocols from Roche Applied Science (USA). The probe was mixed with blocking wheat DNA prepared according to MasoudiNejad et al. (2002), usually in the probe to block ratio of ca. 1:100. Counterstaining was with $1 \%$ propidium iodide. Sequential C-banding- in situ probing were done using the protocol provided by Dr. T. Endo, Kyoto University, Japan. For sequential C-banding- in situ probing, preparations were made in the same fashion as for standard in situ probing, they were C-banded, analyzed and photographed. The slides were then de-stained air dried, and probed using the Masoudi-Nejad et al. (2002) protocol. The cells photographed after C-banding were re-photographed after the in situ probing.

All observations were made under a Zeiss Axioscope 20 equipped with epi-fluorescence, recorded with a SPOT RT Color digital camera (Diagnostic Instruments Inc.), and processed using the SPOT Advanced and Adobe Photoshop CS software to improve contrast and resolution.

\section{Statistics}

Differences in proportions and frequencies of various chromosome types were determined using the standard $\chi^{2}$ test.

\section{Results}

The larger purpose of this study was to generate useful DH lines and no detailed records of the regeneration rates/ success were collected for every attempt or in every season. The total number of green androgenic plants transplanted to soil exceeded 3,500. Weak green plants that did not grow well in test tubes were not transplanted and no record exists of their numbers/proportions. Of the transplants, a certain proportion died before the colchicine treatment and the weakest of the survivors were not colchicine-treated. The estimated overall proportion of weak green plants rejected before colchicine treatment was about $8 \%$, but in different cross combinations it ranged from ca. 2-3\% (in Presto $\times$ Mungis and Krakowiak $\times$ Presto) to 
ca. $70 \%$ in the group of late regenerants in Presto $\times$ NE422T. Mortality due to colchicine was low (about $4.5 \%$ ). Seed set per colchicine-treated plant ranged from a single seed to complete fertility. Complete fertility is probably indicative of spontaneous chromosome doubling in early microspore divisions rather than an effect of colchicine.

In three cycles of androgenesis that included 644 colchicine-treated plants for which fairly detailed counts were made, $157(24.3 \%)$ green plants were deemed to be aneuploid, based on their morphology at flowering. Most of these presumed aneuploids did not set seed. Those that did set seed and had their progeny karyotyped, all were aneuploid. Among plants that appeared normal in $\mathrm{C}_{0}$ and set seed, additional 16 were deemed aneuploid based on their morphology and seed set in $\mathrm{C}_{1}$, and all were found to be aneuploid upon karyotyping. Therefore, the average frequency of aneuploids among all regenerated green plants from all cross combinations must have exceeded $35 \%$. In one of the most recalcitrant combinations, NE422T $\times$ Stan 1, among 72 plants that survived colchicine treatment, $50(69 \%)$ were morphologically abnormal and were considered aneuploids.

A total of 140 plants and lines were karyotyped by Cbanding and in situ probing with total genomic rye DNA. Of these, 67 were $\mathrm{C}_{0}$ plants karyotyped after the colchicine treatment, and 73 were $C_{1}$ and $C_{2}$ generation lines obtained by self pollination of the $\mathrm{C}_{0}$ plants. This was not a random sample. For karyotyping, the $\mathrm{C}_{0}$ plants were selected based on their morphology, such as from among the group of the 157 presumed aneuploids listed above. While all androgenic progenies here were generated from intervarietal $F_{1}$ hybrids and segregation for morphological characters was expected, all deviations from typical triticale morphology were considered suspicious and the plants were karyotyped. In $\mathrm{C}_{1}-\mathrm{C}_{2}$ progenies, in addition to morphology, any reduction in seed set and shriveled seed was deemed suspicious and a reason for karyotyping.

The chromosome numbers of the karyotyped $\mathrm{C}_{0}$ plants were 19, 20, $20+\mathrm{t}$ (where $\mathrm{t}$ denotes a telocentric) or 38, $38+2 \mathrm{t}, 40$ and 42 (Fig. 1a, b). There was also one plant each with 28, 78 and 41 chromosomes. The two groups of
Fig. 1 Sequential C-banding (a) and genomic in situ hybridization (b) on a 20 chromosome $\mathrm{C}_{0}$ androgenic triticale (XTriticosecale Wittmack) plant, nullisomic for rye chromosome 5R. Rye chromatin labeled green; wheat chromatin is red. c C-banded 28 chromosome $\mathrm{C}_{0}$ plant with 21 wheat and 7 rye chromosomes. Wheat chromosomes are: $1 \mathrm{~A}^{\prime}$, $2 \mathrm{~A}^{\prime}, 3 \mathrm{~A}^{\prime}, 4 \mathrm{~A}^{\prime \prime}, 5 \mathrm{~A}^{\prime \prime}, 6 \mathrm{~A}^{\prime \prime}, 7 \mathrm{~A}^{\prime}$, $1 \mathrm{~B}^{\prime \prime}, 2 \mathrm{~B}^{\prime \prime}, 3 \mathrm{~B}^{\prime}, 4 \mathrm{~B}^{\prime \prime}, 5 \mathrm{~B}^{\prime}, 6 \mathrm{~B}^{\prime}$, $7 \mathrm{~B}^{\prime \prime}$ where ' and "denote monosomics and disomics, respectively. d Genomic in situ hybridization with rye genomic probe on a 40 chromosome $\mathrm{C}_{1}$ androgenic triticale plant. Rye chromatin labeled green; wheat chromatin counterstained red: 26 wheat chromosomes, 6 pairs of rye chromosomes and one pair of non-centric wheat-rye translocation (arrowed). The rye chromosome in the translocation is $6 \mathrm{R}$; the wheat segment remains to be unidentified
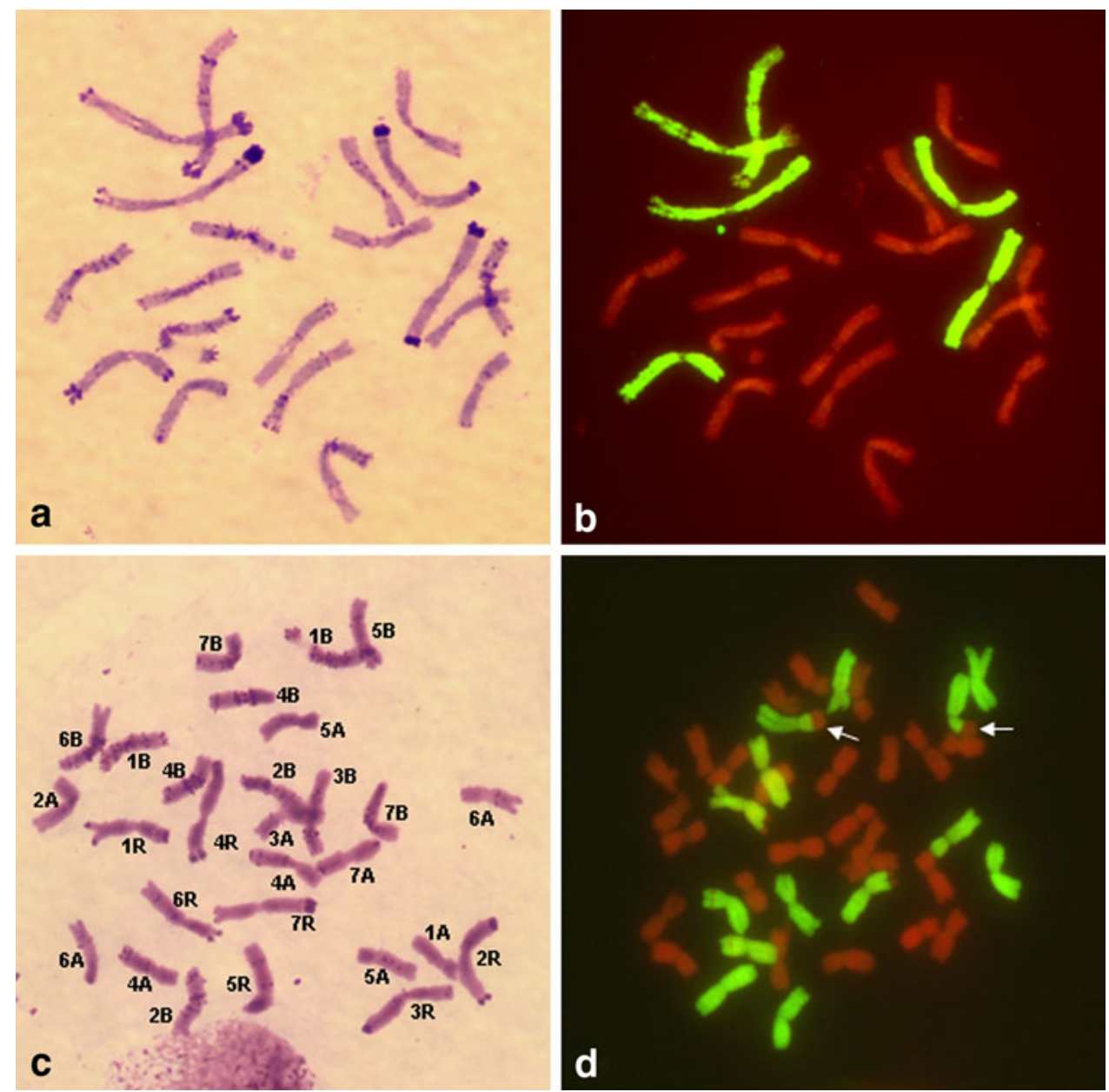
chromosome numbers, $19-20+\mathrm{t}$ and 38-40-42, represent the effect of chromosome doubling. The 42 chromosome plant was nulli5R-tetra7A; the 41 chromosome plant was monosomic $4 \mathrm{~B}$. The origin of the 28 and 78 chromosome plants could not be ascertained: the 28 chromosome plant had 7 monosomic and 7 disomic wheat chromosomes and 7 monosomic rye chromosomes (Fig. 1c). The 78 chromosome plant was tetrasomic for all chromosomes except $2 \mathrm{R}$, for which it was nullisomic, and $4 \mathrm{~B}$ for which it was disomic.

Among $\mathrm{C}_{1}-\mathrm{C}_{2}$ plants karyotyped, chromosome numbers were $38,38+2 \mathrm{t}, 40,40+2 \mathrm{t}, 42$ and 44 . One plant was somatically unstable. Among 42 chromosome plants, 16 were karyotypically normal, one was a compensating nullitetrasomic (nulli $1 \mathrm{~B}$ tetra $1 \mathrm{R}$ ), two were nulli-tetrasomics of random chromosomes (nulli7A-tetra 1A; nulli1B-tet$\mathrm{ra} 2 \mathrm{R}$ ) and one was a homozygote for a wheat-rye translocation involving rye $6 \mathrm{R}$ and an unidentified wheat segment (Fig. 1d). The 44 chromosome plant had four pairs of chromosomes in group 1:1A, 1B, 1R and an engineered 1R (Lukaszewski 2006).

Most aneuploids were nullisomics, followed by ditelosomics (Table 1); the former were more frequent in $\mathrm{C}_{0}$; the latter in $\mathrm{C}_{1}-\mathrm{C}_{2}$. Several acrocentrics were also present; for simplicity they are included among telosomics as both are products of univalent misdivision. There were 15 identifiable wheat-rye translocations (Fig. 1d). Essentially all aneuploids were hypoploids; only two hyperploids (44 and 78 chromosomes) were present. Among 141 chromosomes involved in aneuploidy, each genome and each chromosome was represented at least once (Table 1). Overall, in all progenies karyotyped, rye chromosomes were about four times more likely to be involved in aneuploidy than wheat chromosomes. However, there were clear differences between the $\mathrm{C}_{0}$ and $\mathrm{C}_{1}-\mathrm{C}_{2}$ generations. Most $\mathrm{C}_{0}$ plants karyotyped were nullisomics for one or more rye chromosomes and chromosome $5 \mathrm{R}$ was the most frequently involved, followed by $2 \mathrm{R}$. Nullisomy for wheat chromosomes was infrequent. Among all $\mathrm{C}_{0}$ aneuploids, only 12 cases involved a wheat chromosome, as opposed to 53 for rye (the 28 and 72 chromosome plants are not included in this total as it is not clear how to interpret their chromosome constitutions). In $\mathrm{C}_{1}-\mathrm{C}_{2}$ the numbers of rye and for wheat chromosomes that contributed to aneuploidy were almost equal (37 vs. 39, respectively) with similar low frequencies for each chromosome present, except for $1 \mathrm{~B}$ which was the most frequently involved wheat chromosome in both classes of progeny.

SDS-PAGE screening was limited to combination involving engineered chromosomes $1 \mathrm{R}$ (Lukaszewski 2006). It was capable of verifying the chromosome constitution of only the group-1 chromosomes. Among 752 lines screened, 19 were identified as group-1 aneuploids, based on the presence-absence, and the intensity of glutenin subunits specific to individual chromosomes and chromosome arms. For example, absence of the 1Bencoded high molecular weight glutenins and gliadins (loci $G l u-B 1$ and $G l i-B 1$, respectively) with the concomitant increase in the intensity of the rye-encoded secalins Sec-1 and Sec-3, was interpreted as nullisomy 1B-tetrasomy $1 \mathrm{R}$. These19 identified aneuploids included nullisomics $1 \mathrm{R}$ or 1B, nulli1R-tetra1B and nulli1B-tetra1R. In all instances, karyotyping confirmed chromosome constitutions predicted by SDS-PAGE.

The large difference in proportions of wheat and rye chromosomes involved in aneuploidy between $\mathrm{C}_{0}$ and

Table 1 The involvement of individual chromosomes in aneuploidy among $140 \mathrm{C}_{0}, \mathrm{C}_{1}$, and $\mathrm{C}_{2}$ haploids and doubled haploids of hexaploid triticale (XTriticosecale Wittmack) generated by androgenesis

\begin{tabular}{|c|c|c|c|c|c|c|c|c|}
\hline \multirow[t]{2}{*}{ Genome } & \multicolumn{7}{|c|}{ Homoeologous group } & \multirow[t]{2}{*}{ Total } \\
\hline & 1 & 2 & 3 & 4 & 5 & 6 & 7 & \\
\hline \multirow[t]{3}{*}{ A } & $N=3$ & $N=1$ & $N=1$ & $N=3$ & $N=1$ & $N=2$ & $N=3$ & $N=14$ \\
\hline & Dt $=1$ & $\mathrm{Dt}=1$ & $\mathrm{Dt}=0$ & $\mathrm{Dt}=0$ & Dt $=1$ & $\mathrm{Dt}=1$ & $\mathrm{Dt}=0$ & $\mathrm{Dt}=4$ \\
\hline & $T=2$ & $T=0$ & $T=0$ & $T=0$ & $T=0$ & $T=0$ & $T=2$ & $T=4$ \\
\hline \multirow[t]{3}{*}{ B } & $N=16$ & $N=0$ & $N=0$ & $N=2$ & $N=0$ & $N=2$ & $N=1$ & $N=21$ \\
\hline & Dt $=1$ & $\mathrm{Dt}=0$ & $\mathrm{Dt}=0$ & $\mathrm{Dt}=0$ & $\mathrm{Dt}=0$ & $\mathrm{Dt}=2$ & $\mathrm{Dt}=0$ & $\mathrm{Dt}=3$ \\
\hline & $T=1$ & $T=0$ & $T=1$ & $T=0$ & $T=1$ & $T=0$ & $T=0$ & $T=3$ \\
\hline \multirow[t]{3}{*}{$\mathrm{R}$} & $N=7$ & $N=15$ & $N=7$ & $N=6$ & $N=24$ & $N=4$ & $N=6$ & $N=69$ \\
\hline & Dt $=4$ & $\mathrm{Dt}=2$ & $\mathrm{Dt}=1$ & $\mathrm{Dt}=2$ & $\mathrm{Dt}=3$ & $\mathrm{Dt}=3$ & $\mathrm{Dt}=0$ & $\mathrm{Dt}=15$ \\
\hline & $T=3$ & $T=1$ & $T=2$ & $T=0$ & $T=1$ & $T=1$ & $T=0$ & $T=8$ \\
\hline \multirow[t]{3}{*}{ Total } & $N=26$ & $N=16$ & $N=8$ & $N=11$ & $N=25$ & $N=8$ & $N=10$ & $N=104$ \\
\hline & Dt $=6$ & $\mathrm{Dt}=3$ & $\mathrm{Dt}=1$ & $\mathrm{Dt}=2$ & $D t=4$ & $\mathrm{Dt}=6$ & $\mathrm{Dt}=0$ & $\mathrm{Dt}=22$ \\
\hline & $T=6$ & $T=1$ & $T=3$ & $T=0$ & $T=2$ & $T=1$ & $T=2$ & $T=15$ \\
\hline
\end{tabular}

$N$ nullisomics, $D t$ Ditelosomics/telosomics, $T$ translocations or other unidentified modifications of a chromosome 
$\mathrm{C}_{1}-\mathrm{C}_{2}$ was a consequence of selection for fertility. Notwithstanding hybrid origin of the androgenic plants analyzed here, two distinct phenotypes were evident in each batch of recovered progenies (Fig. 2). One had thin stems, very narrow leaves at a high angle to the stems (usually close to $90^{\circ}$ or drooping down in later stages of plant growth). This type was named "Narrow". The other type had wide leaves twisted along their length, stems much thicker than normal, and the plants tended to be lighter green with yellow streaks along the veins (Fig. 2). This type was named "Curly". While the frequency of Curly remained relatively constant at about $2-4 \%$ in all batches of plants produced regardless of the cross combination, the frequency of Narrow varied from one combination to another, and with time spent in culture. It was especially prevalent in the most recalcitrant combination, Presto $\times$ NE422T, where among 84 green plants produced in the spring of 2008, 40 (47.6\%) had that distinct morphology. However, in the first batch of 48 plants obtained (the shortest time in culture) 17 were Narrow (35.4\%); in the second batch of 36 plants (the longest time in culture), 23 were Narrow (63.8\%). The difference in proportions of Narrow in the two batches was significant $\left(\chi^{2}=6.69, p<0.01\right)$. A similar effect was also observed in Mungis $\times$ Stan 1 , where among 56 early obtained green plants none were Narrow, but among 93 lateobtained plants 10 were Narrow. For a comparison, in a combination Presto $\times$ Krakowiak which was quite amenable to androgenesis, in the first batch of 125 plants 3 were Narrow (2.4\%); in a batch of 175 plants generated four weeks later, eight $(4.6 \%)$ were Narrow. The only exception to the relatively stable frequency of Curly across all combinations was NE422T $\times$ Stan 1, where it constituted $24 \%$ of all plants in the first (earliest) batch of green plants produced. However, only two Curly plants were present in the second batch of plants produced $(4.4 \%)$ so that the overall frequency of Curly in this combination was not much different from the average.

All plants with the Narrow phenotype were nullisomics $5 \mathrm{R}$; at times nullisomy $5 \mathrm{R}$ was accompanied by other aberrations. Among 13 plants with the Curly phenotype tested here, 12 were nulli-2R and one was nulli-2A. One double nullisomics $2 \mathrm{R}$ and $5 \mathrm{R}$ had the Narrow phenotype. Plants with the Narrow phenotype were sterile regardless of their ploidy level; only one plant set three seeds and hence its chromosome constitution could be confirmed in the subsequent generation. Plants with the Curly phenotype were capable of setting several seeds each. Two such progenies were re-karyotyped and their nullisomy $2 \mathrm{R}$ was confirmed. It was clear that nullisomy for rye chromosomes had a far greater impact on seed set than that for wheat chromosomes, and the sterility barrier eliminated most of rye nullisomics between $\mathrm{C}_{0}$ and $\mathrm{C}_{1}$, changing the proportions of aneuploidy for wheat and rye chromosomes in the two generations. Most aneuploids in $\mathrm{C}_{1}$ and $\mathrm{C}_{2}$ involved ditelosomy and chromosome translocations.

While most material analyzed here was pre-selected based on plant morphology, seed set and seed quality, one hybrid combination, Presto $\times$ Mungis, was studied in its entirety in its second generation. No colchicine was used so this population of $\mathrm{DH}$ is composed strictly of plants resulting from spontaneous chromosome doubling. Of the 1200 green plants regenerated, 207 plants were fully fertile while 27 were partially fertile. Upon self-pollination, progenies of all these plants were grown and subjected to
Fig. 2 Two most frequent phenotypes among aneuploid $\mathrm{C}_{0}$ plants recovered via androgenesis in hexaploid triticale (XTriticosecale Wittmack): on the left Nulli $2 \mathrm{R}$ ('Curly' phenotype); on the right Nulli 5R ('Narrow' phenotype)
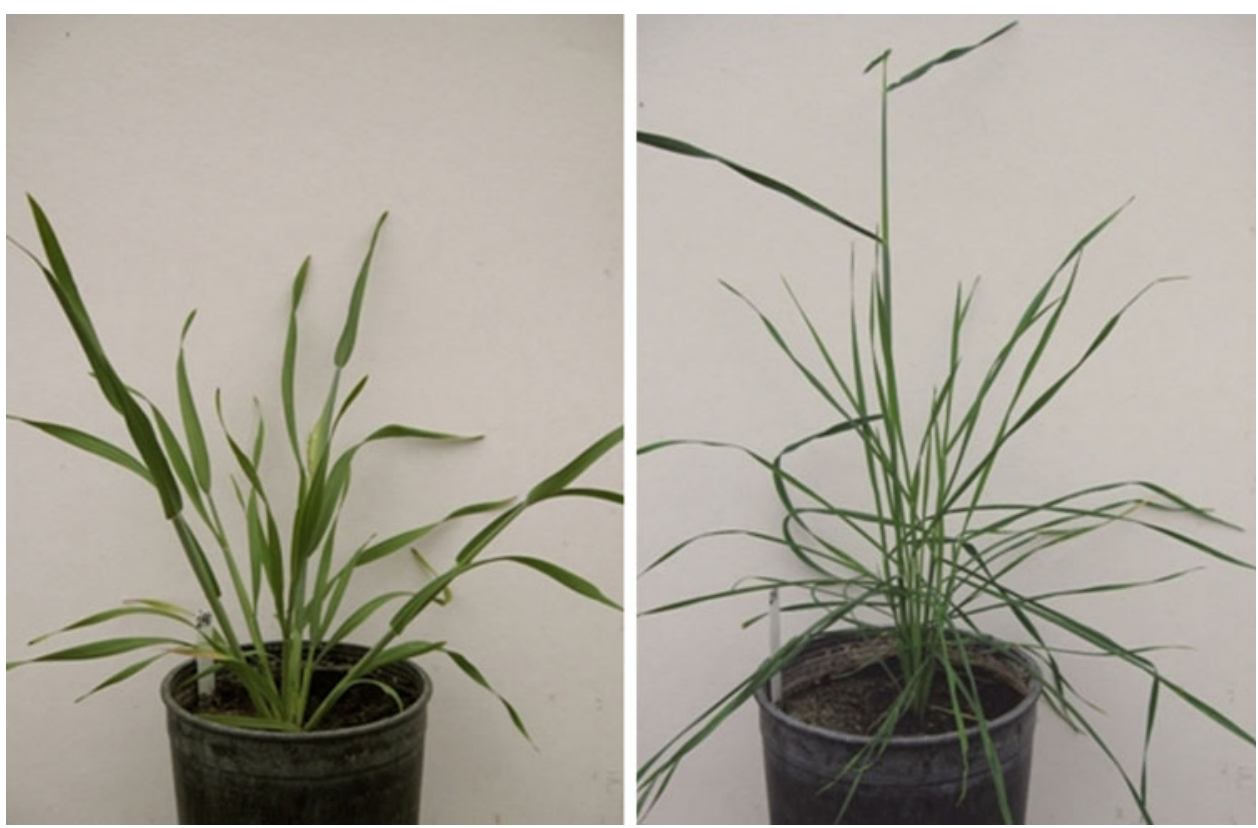
flow cytometry to determine their DNA content. No reliable reading was obtained for eight lines; all eight lines were morphologically normal and set normal seed. Three of these were karyotyped and found to be euploid. Of the remaining 226 lines, the FC flagged 19 as aneuploid because the peaks of their DNA contents distribution clearly separated from the distribution peaks of the parents, used as standards. Among these 19 lines, 17 were morphologically normal and set normal seed while two appeared aneuploid. Both morphologically aneuploid lines were confirmed to be aneuploid upon karyotyping (both had 40 chromosomes; one was nullisomic $4 \mathrm{~A}$ and one had a wheat-rye translocation that appeared to be $1 \mathrm{RS} .1 \mathrm{AL}$. Of the nine lines tested that were flagged by FC as aneuploid but which were morphologically normal, cytologically all were euploid. Among 207 lines that appeared euploid based on the FC, two were morphologically abnormal and were cytologically confirmed to be aneuploid. Hence, plant's morphology was a more reliable indicator of the chromosome status of the DH lines than flow cytometry.

MI pairing in $F_{1}$ hybrids

Four $\mathrm{F}_{1}$ hybrids (Presto $\times$ NE422T, NE422T $\times$ Stan 1 , Mungis $\times$ Stan 1, and Mungis $\times$ Krakowiak) were tested for their MI chromosome pairing and specifically, for the frequency of univalents per pollen mother cell (PMC). While each B-genome univalent was identified, no attempts were made to identify individual A-genome univalents; therefore, all wheat univalents are pooled together. Because of a considerable C-band polymorphism between the parents of the tested hybrids, individual rye chromosomes could not be identified with confidence in each PMC scored. Therefore, rye univalents were classified into two groups: a group of four chromosomes with low arm ratios and usually both telomeres with heavy telomeric bands (1R, 2R, 3R and 7R), and a group of three chromosomes with high arm ratios and normally only one banded telomere (4R, 5R and 6R).

Among the four hybrids tested, three were relatively stable with the average number of univalents per PMC averaging between 1.53 and 1.88 per PMC and proportion of PMC without univalents ranging from 36 to $46 \%$, and one, NE422T $\times$ Stan 1, was far more unstable, with 3.01 of univalents per PMC and only $13.3 \%$ of PMCs with complete chromosome pairing (Table 2). In all instances, rye chromosomes were at least four times more likely to contribute to univalency than wheat chromosomes (based on the genome ratios). Wheat genomes $\mathrm{A}$ and $\mathrm{B}$ were equally likely to contribute to univalency; however, among the B-genome chromosomes, chromosome 1B contributed about one half of all univalents of the genome, followed by 5B. Among rye chromosomes, in the three more stable hybrids, the four almost median chromosomes with both arms normally carrying heavy telomeric bands (1R, 2R, 3R and $7 \mathrm{R})$ were significantly $(p<0.01)$ more likely to be univalent than the group of three chromosomes with high arm ratios and only one large telomeric C-band (4R, 5R, $6 \mathrm{R})$. As far as it could be established, among the group of chromosomes with high arm ratios (4R, 5R, 6R), each chromosome contributed to univalency in similar proportions, and these frequencies were lower than the average for the entire genome. In NE422T $\times$ Stan 1 the probability of chromosomes from the two groups contributing to univalency did not significantly deviate from random $\left(\chi^{2}=2.3828\right)$ but among the group of $4 \mathrm{R}, 5 \mathrm{R}$ and $6 \mathrm{R}$ chromosomes, $5 \mathrm{R}$ appeared to be present as a univalent with a higher frequency than the other two.

\section{Discussion}

The main purpose of the project was to generate populations of DH triticale lines for genetic mapping. Aneuploidy came into focus only when it was evident how seriously it affected the efficiency of the approach. Still, the issue of aneuploidy was addressed in a manner that would not impact the primary goal of the project. A systematic approach to the issue of aneuploidy would have required different handling of the material to satisfy all requirements of cytology and likely would have negatively affected the chromosome doubling rates, hence the recovery of the $\mathrm{DH}$

Table 2 Mean frequencies of univalents in MI of meiosis in four $\mathrm{F}_{1}$ hybrids of winter triticale (XTriticosecale Wittmack)

\begin{tabular}{|c|c|c|c|c|c|c|}
\hline \multirow[t]{3}{*}{ Combination } & \multirow{3}{*}{$\begin{array}{l}\text { Number of } \\
\text { PMCs scored }\end{array}$} & \multirow{3}{*}{$\begin{array}{l}\text { Percent of PMCs } \\
\text { with full pairing }\end{array}$} & \multicolumn{4}{|c|}{ Univalents per PMC } \\
\hline & & & \multirow[t]{2}{*}{ Wheat } & \multicolumn{2}{|l|}{ Rye } & \multirow[t]{2}{*}{ Tota } \\
\hline & & & & Low arm ratio & High arm ratio & \\
\hline Presto $\times$ NE422T & 100 & 46.0 & 0.42 & 0.86 & 0.36 & 1.64 \\
\hline $\mathrm{NE} 422 \mathrm{~T} \times \mathrm{Stan} 1$ & 150 & 13.3 & 1.04 & 1.04 & 0.93 & 3.01 \\
\hline Mungis $\times$ Stan 1 & 100 & 36.0 & 0.64 & 0.86 & 0.34 & 1.88 \\
\hline Mungis $\times$ Krakowiak & 150 & 45.3 & 0.28 & 0.82 & 0.43 & 1.53 \\
\hline
\end{tabular}

"Low arm ratio" rye chromosomes are $1 \mathrm{R}, 2 \mathrm{R}, 3 \mathrm{R}$ and $7 \mathrm{R}$; "high arm ratio" chromosomes are 4R, 5R and 6R 
lines. While some data presented here may not be representative for entire populations, generations or types of hybrids, chromosome identities and the types of aberrations observed do not appear to have been affected by the method in which the data were collected. The overall frequencies of aneuploids recovered here were within the ranges observed in hexaploid triticale before (Charmet et al. 1986; Ryöppy 1997). Moreover, the proportions of wheat and rye chromosomes contributing to aneuploidy as well as the identities of individual rye chromosomes involved were similar to the random sample of Charmet et al. (1986).

Plants for karyotyping here were selected based on their morphology, seed set and high proportions of shriveled seed. As androgenic progenies these plants had to be either haploid or DHs; any deviation from the standard chromosome number and constitution always resulted in nullisomy or tetrasomy for a specific chromosome or chromosome arm; translocations, if present, were always disomic. This made identification of aneuploids simple and reliable. Monosomics, telo-disomics (a pair of chromosomes represented by one normal and one telosomic) and translocations heterozygotes would have been much more difficult to identify by morphology. However, all triticale monosomics observed so far (Lukaszewski 1990) had reduced seed set and most seed was shriveled so at least the monosomics and telo-disomics could have been identified if not by plant morphology then certainly by seed characteristics at harvest.

Aneuploidy among DHs is a fairly common phenomenon, regardless of the method of regeneration, be it androgenesis (Metz et al. 1988), gynogenesis (Aleza et al. 2009) or wide hybridization (Rines and Dahleen 1990). It appears to be a particularly important problem in androgenesis (Dogramaci-Altuntepe et al. 2001). The mechanisms responsible for alterations in chromosome number, structure, and constitution among the regenerated progenies are not entirely clear. The factors that may contribute to the aneuploidy include genotypic differences among donor plants, stress applied to plants or cut tillers before plating, culture conditions, media composition (e.g. growth regulators), duration of the culture phase, the callus stage, colchicine treatment (Logue 1996) but first and foremost, the characteristics of the starting material itself.

Aneuploidy in the $\mathrm{C}_{0}$ generation may have two origins: either aneuploid microspores develop into haploids or chromosome(s) are lost or broken during microspore development, as discussed in great detail by Logue (1996). In other words, it is either a consequence of imperfect starting material (the microspores) or of an imperfect process of sporophytic development of a microspore in the in vitro culture. On the other hand, aneuploidy among $\mathrm{C}_{1}$ and later generations may have two sources: a carry over from $\mathrm{C}_{0}$, if an aneuploid is fertile enough to produce seed, or be a consequence of irregular chromosome pairing in $\mathrm{C}_{0}$ or later, resulting in aneuploid gametes that produce aneuploid progeny. To simplify further discussion, aneuploids generated either from aneuploid microspores or by chromosome instability during androgenesis itself will be referred to in this text as primary aneuploidy; aneuploidy due to meiotic instability of regenerated plants will be called secondary aneuploidy.

A clear discrimination between the two possible origins of primary aneuploids cannot be easily made as their consequences are essentially identical: once the chromosome number is doubled, a plant becomes disomic for the chromosome aberration (absence or extra dose of a chromosome or chromosome arm, translocation). If the type of aberration permits seed set, all progeny shares the same phenotype and the same chromosome aberration (excepting the possibility that the original aneuploidy promotes secondary aneuploidy). The only exception here would be chromosome doubling by a somatic fusion of two nuclei, each with different chromosome numbers/constitutions sometime during the process of androgenesis.

Fusion of sister nuclei in the early divisions of microspores pushed into the sporophytic pathway of development are well documented and appear responsible for the frequent occurrence of spontaneously DHs (Kasha et al. 2001; Shim et al. 2006). The most plausible explanation for chromosomal differences between two sister nuclei (that is, nuclei within a single cell, produced by standard mitosis) is chromosome nondisjunction, in effect doubling the number of copies of this specific chromosome in one daughter nucleus, while leaving its sister nucleus deficient for one copy. Fusion of such sister nuclei automatically restores normal chromosome constitution, masking the nondisjunction event. On the other hand, fusions of non-sister/ different nuclei, if technically possible, would be more likely to occur in meiocytes where thick cell walls are not a barrier, and would have a chance of not only producing aneuploids but would almost always produce heterozygotes. Such heterozygotes would be quite unsuitable for the general purposes for which DH lines are made, and would be readily detectable upon the first seed increase by segregation of the characteristics of the two parents. They would also be difficult to distinguish from regenerants from somatic tissues of the anther. Heterozygous regenerants did appear with low frequencies (below 1\%, data not shown) among the plants produced here. They seemed better explained by imperfect isolation during flowering and resulting spontaneous outcrossing of the $\mathrm{C}_{0}$ plants rather than regeneration from somatic tissues or by fusion of non-sister nuclei. Aneuploids produced in the process of outcrossing would most likely create heterozygous aberrations, such as monosomics, telocentric plus complete 
chromosome and translocation heterozygotes. Disomic aberrations, while statistically possible, would be quite unlikely. Essentially all aberrations observed here were disomic.

Somatic chromosome instability following nuclear fusions, or after induced chromosome doubling, would result in single-dose aberrations (as opposed to disomy/homozygosity as in case of all pre-doubling events). Aneuploids produced in this fashion would be difficult to detect by morphology; after harvest and in the subsequent generations they would be practically indistinguishable from secondary aneuploids resulting from imperfect chromosome pairing in the $\mathrm{C}_{0}$ plants. However, one line was observed here that raised suspicion of a possible fusion of non-sister nuclei producing an aneuploid. Most of its progeny appeared aneuploid and segregated for many morphological characters. However, its origin from somatic tissue, combined with a chromosome loss during regeneration, cannot be ruled out.

Meiotic instability in $\mathrm{C}_{0}$ or later generation is most likely to produce a single-dose chromosome aberration (monosomy, trisomy, telosomy, translocation heterozygote) and in individual progeny plants while a majority of progeny would maintain their uniform phenotype. This feature was used to discriminate between the primary and secondary aneuploidy. Secondary aneuploidy, that is the frequency of aneuploids generated by imperfect chromosome pairing in $\mathrm{C}_{0}$ or $\mathrm{C}_{1}$, was estimated here at ca. 2.0-3.6\%, in different seasons and different combinations. The estimate is based on the frequency of single plants deviating from the morphology typical for a line in the $\mathrm{C}_{1}$ seed increase.

Observations of the MI chromosome pairing confirmed considerable meiotic instability of triticale hybrids (Struss and Röbbelen 1989). In the most stable hybrid analyzed, Presto $\times$ NE422T, only $46 \%$ of PMCs had all chromosomes paired with no univalents present; the same parameter for the least stable hybrid, NE422T $\times$ Stan 1, was $13.3 \%$ (Table 2). The numbers of univalents, when present, ranged from 2 to 10 per PMC. Most univalents were from the rye genome. This suggests that aneuploidy among regenerants is primarily a consequence of a high frequency of aneuploid microspores in the plated material. Apparently, aneuploidy does not preclude a microspore from the switch to sporophytic development under culture conditions. Given the proportions of PMCs with complete chromosome pairing and proportions of euploid and aneuploid regenerants in this study, it did not appear that aneuploidy per se predisposed microspores to the switch to the sporophytic pathway of development. In the meiotically more stable combinations, the proportions of aneuploids were lower; conversely, in NE422T $\times$ Stan 1 with the lowest proportion of PMCs with complete chromosome pairing, aneuploids greatly outnumbered euploids among the regenerants. Moreover, higher frequencies of aneuploids among regenerants may be related to the general amenability of a combination to androgenesis. In the two most recalcitrant combinations, Presto $\times$ NE422T and $\mathrm{NE} 422 \mathrm{~T} \times$ Stan 1 , aneuploids predominated among the regenerants, frustrating even more all attempts to create populations of DH lines of sufficient sizes.

Among aneuploid microspores producing regenerants, the possible role of chromosome $5 \mathrm{R}$ is intriguing. Among $67 \mathrm{C}_{0}$ aneuploids analyzed, in 52 cases a rye chromosome was involved, but 23 of these cases involved chromosome $5 R$. This is very similar to the proportion of 40 aneuploids $5 \mathrm{R}$ among the total of 83 rye chromosomes involved in aneuploidy in androgenic triticales in the study of Charmet et al. (1986). In three of the four hybrids analyzed at MI, there were no indications that chromosome $5 \mathrm{R}$ was more prone to pairing failure and hence, contributed to aneuploidy in an unduly high proportion. This suggests a possible role of chromosome $5 \mathrm{R}$ in the green plant regeneration. Both hybrids involving NE422T responded poorly to culture conditions, and both generated a large proportion of aneuploids. One hybrid was relatively stable in meiosis, the other was unstable. A majority of green plants recovered in both combinations were aneuploid, most of them were nulli $5 \mathrm{R}$, and the proportion of nulli $5 \mathrm{R}$ increased with time spent on the induction medium. The overall low yield of regenerants in these combinations was a consequence of a very low proportion of green plants; there were no obvious differences in the ability to form embryos and embryogenic structures (data not shown). It is not clear at this point what effect an absence of a specific chromosome may have on the ability to form green plants. It is also unclear if the increase in $5 \mathrm{R}$ nullisomics among green plants over time reflects a delayed reaction of the 5Rdeficient microspores to culture conditions or a gradual loss of chromosomes $5 \mathrm{R}$ during different stages of the regeneration process. As there are no indications of an unusually high proportion of $5 \mathrm{R}$ deficient microspores in the two hybrids, such microspores were presumably selected for during androgenesis, suggesting some involvement of $5 \mathrm{R}$ in the regenerations process. Chromosome $5 \mathrm{R}$ was not identified as a carrier of QTLs for androgenic response in hexaploid triticale by Gonzalez et al. (2005) and cv. Presto was one of the parental lines in that study. On the other hand, selection for specific chromosome constitutions among microspores has been demonstrated in Brassica hybrids (Nelson et al. 2009) so it cannot be ruled out in these materials.

If the two distinct aneuploid phenotypes, Curly and Narrow (Nulli 2R and Nulli 5R, respectively) are eliminated from consideration (essentially all are eliminated between $\mathrm{C}_{0}$ and $\mathrm{C}_{1}$ by sterility), rye chromosomes still contributed to aneuploidy about twice as frequently as 
wheat chromosomes, on a per chromosome basis, and this parallels the contribution of rye and wheat chromosomes to univalency in MI. It is therefore possible that the primary cause of high aneuploidy among DHs of triticale relates directly to incomplete chromosome pairing in MI and resulting aneuploid microspores. The most frequent aberrations here were nullisomy and tetrasomy; however, ditelosomy and most translocations can also be traced back to univalency in MI. Paired chromosomes do not misdivide to form telocentrics or centric translocations. In several instances, non-centric translocations were observed (Fig. 1d), but they were a clear minority. The mechanism by which they originated is unknown. On the one hand, the meiotic preparations were analyzed carefully enough to exclude a significant chance of homoeologous pairing. On the other hand, non-centric breakage of univalents and subsequent fusion has been noted (Lukaszewski 1997) so it appears more likely that the observed non-centric translocations resulted from chromosome breakage and fusion rather than by homoeologous recombination. Exceptions to all these considerations are three compensating nullitetrasomics in homoeologous group 1. All of them involved engineered chromosomes 1R; these chromosomes are sixbreakpoint wheat-rye translocations (Lukaszewski 2006). The nulli-tetrasomics observed here likely arose by multivalent pairing of the engineered $1 \mathrm{R}$ with their standard wheat and rye precursors, and uneven segregation from multivalents in anaphase I. These nulli-tetrasomics were practically indistinguishable from euploids, both by morphology and by seed characteristics.

Secondary aneuploids were present in the analyzed material. This was judged by the frequency of single plants deviating from the standard phenotype of any given $\mathrm{DH}$ line. Among 844 and 488 plants scored for this feature in two different growing seasons, 21 and 14 were classified as aneuploid, respectively, for the frequencies of 2.5 and $2.9 \%$, respectively. Of the first 21 , six were tested cytologically and all were monosomic for various chromosomes. The $2.6 \%$ frequency of secondary aneuploidy among the DH lines is within the $2-3 \%$ range of aneuploid frequency assessed visually for the parental lines used in the study, and is at the lower end of perhaps the most recent data on aneuploidy in commercial hexaploid winter triticales (2.0-7.0\%; Lelley 1992) but considerably below the ranges observed by Struss and Röbbelen (1989) among $F_{1}$ hybrids of triticale. These observations give no reason to suspect that the DH lines of hexaploid triticale will be less stable than the parental lines. On the other hand, if meiotic instability of triticale hybrids reflects differences in genetic systems of chromosome pairing control among parental lines, segregation among progeny is to be expected with an overall wider range of variation for various indices of chromosome paring than among the parents.
Flow cytometry is often used to assess chromosome constitutions of regenerated DH progenies (Hu and Kasha 1997; Kim et al. 2003). This approach suffers certain methodological weakness in that any variation in the DNA contents between the parents may generate a range of variation among the regenerants that can be confused with aneuploidy. Among androgenic progenies from the Lolium $\times$ Festuca hybrids, Guo et al. 2005 found a 5-7\% difference in the nuclear DNA contents. Given that these regenerants had 14 or 28 chromosomes, a $7 \%$ difference could easily be interpreted as nullisomy while in fact it may only reflect a general difference of the DNA contents between the two parents. The same may hold true for hexaploid triticale. Triticale is known for considerable differences in the amount of telomeric chromatin present on rye chromosomes. Telomeric C-bands in rye occupy about $12 \%$ of the total chromosome length and may contain as much as $18 \%$ of the total nuclear DNA (see Lukaszewski and Gustafson 1987). Given the amounts of DNA per A, B and $\mathrm{R}$ genomes, $18 \%$ difference in the amount of rye DNA present would be equivalent to ca. $7 \%$ difference in the total nuclear DNA contents, an equivalent to the absence or an extra dose of three chromosomes. Given that any two triticales may carry similar amounts of DNA and still differ in the presence/absence of certain heterochromatic blocks, flow cytometry results have to be interpreted with extreme caution and as a rule should not be used to asses the aneuploidy rates, or the general chromosome stability of the regenerated material (Kim et al. 2003). In this study, there was no significant difference in the DNA contents between the two parents in a cross (Presto and Mungis) and yet, there was $11.3 \%$ difference in the DNA contents among tested DH progeny (data not shown), and these differences did not correlate with aneuploidy.

While a majority of aneuploids observed here can be explained as a consequence of pairing failure in MI of meiosis of the donor plants, there were also some aneuploids with chromosome constitution that defied all efforts at explanation. Among them was a plant with 28 chromosomes where seven chromosomes were present in pairs and 14 were present in single dosage (Fig. 1c). Also interesting in this context was the 78 chromosome plant (tetrasomic for all chromosomes except nullisomy $2 \mathrm{R}$ and disomy $4 \mathrm{~B}$ ). It could not have originated by two rounds of chromosome doubling unless an assumption is made of somatic chromosome loss (here 4B) between the two rounds of doubling. Individuals with similarly impossible to explain origins were noted among androgenically derived progenies of Festuca arundinacea x Lolium multiflorum hybrids (Zwierzykowski et al. 1998) or haploids generated from tetraploid Festulolium (Kopecky et al. 2005). Apparently, there are some yet unknown pathways by which aneuploids can be generated. 
It is clear that aneuploidy is a very serious problem in androgenesis of hexaploid triticale. In most crops where androgenesis is used, regular meiotic pairing provides for a chromosome constitution of the microspores. In the absence of structural chromosome differences between the parents of a hybrid, any deviation from the standard karyotype among the regenerants can be attributed to chromosome instability while in culture (Kudirka et al. 1983). Still, in crops with regular meiosis such as wheat and rice, 11.1 and $10.2 \%$ aneuploids among regenerants have been detected, respectively (Chu et al. 1985; Kim et al. 2003). In triticale, with its inherent tendency to univalency, especially in $\mathrm{F}_{1}$ hybrids (Struss and Röbbelen 1989), there is an ample supply of aberrant microspores, and at least some data presented here can be interpreted as indicative of selection for such microspores at some stage in the process of androgenesis. While chromosomal abnormalities are noted in numerous crops to which androgenesis has been successfully applied (Forster and Thomas 2005), its frequency in triticale is such that any population of the DH lines must be produced considerably larger than the minimum required, to compensate for the aneuploids. Unfortunately, as experience here shows, aneuploidy appears to be the most frequent in the most recalcitrant combinations. This is perhaps because it eliminates, with some frequency, chromosomes that carry genetic loci that prevent the switch from the gametophytic to the sporophytic microspore. While aneuploids generated by androgenesis could potentially be used for some genetic experiments such as marker allocation to chromosomes, by and large they are an additional burden on the already cumbersome method and may tip the scale toward unprofitability.

Open Access This article is distributed under the terms of the Creative Commons Attribution Noncommercial License which permits any noncommercial use, distribution, and reproduction in any medium, provided the original author(s) and source are credited.

\section{References}

Aleza P, Juárez J, Hernández M, Pina JA, Ollitrault P, Navarro L (2009) Recovery and characterization of a Citrus clementina Hort. ex Tan. 'Clemenules' haploid plant selected to establish the reference whole Citrus genome sequence. BMC Plant Biol 9:110. doi: 10.1186/1471-2229-9-110, http://www.biomedcentral.com/1471$2229 / 9 / 110$

Brzezinski W, Lukaszewski AJ (1998) Allelic variation at the Glu-1, $\mathrm{Sec}-2$, and Sec-3 loci in winter triticale. In: Proceedings of the Fourth International Triticale Symposium, Red Deer, Canada, pp 6-12

Charmet G, Bernard S, Bernard M (1986) Origin of aneuploid plants obtained by anther culture in triticale. Can J Genet Cytol 28:444-452

Chu Q, Zhenhua Z, Yuanhua G (1985) Cytogenetical analysis on aneuploids obtained from pollenclones of rice (Oryza sativa L.). Theor Appl Genet 71:506-512
Dogramaci-Altuntepe M, Peterson TS, Jauhar PP (2001) Anther culture-derived regenerants of durum wheat and their cytological characterization. J Hered 92:56-64

Forster BP, Thomas WTB (2005) Doubled haploids in genetics and plant breeding. In: Janick J (ed) Plant Breeding Reviews, vol 25. Wiley, Hoboken, pp 57-88

Forster BP, Heberle-Bors E, Kasha KJ, Touraev A (2007) The resurgence of haploids in higher plants. Trends Plant Sci 12(8):368-375

Galbraith DW, Harkins KR, Maddox JM, Ayres NM, Sharma DP, Firoozabady E (1983) Rapid flow cytometric analysis of the cell cycle in intact plant tissues. Science 220:1049-1051

Giraldez R, Cermeno MC, Orellana J (1979) Comparison of Cbanding pattern in the chromosomes of inbred lines and open pollinated varieties of rye. Z Pflanzenzucht 83:40-48

Gonzalez JM, Muniz LM, Jouve N (2005) Mapping of QTLs for androgenetic response based on a molecular genetic map of XTriticosecale Wittmack. Genome 48:999-1009

Guo Y, Mizukami Y, Yamada T (2005) Genetic characterization of androgenic progeny derived from Lolium perenne $\times$ Festuca pratensis cultivars. New Phytol 166:455-464

Hu T, Kasha KJ (1997) Performance of isolated microspore-derived double haploids of wheat (Triticum aestivum L.). Can J Plant Sci 77:549-554

Kasha KJ, Hu TC, Oro R, Simion E, Shim YS (2001) Nuclear fusion leads to chromosome doubling during mannitol pretreatment of barley (Hordeum vulgare L.) microspores. J Exp Bot 52(359):1227-1238

Kim KM, Baenziger PS, Rybczynski JJ, Arumuganathan K (2003) Characterization of ploidy levels of wheat microspore-derived plants using laser flow cytometry. In Vitro Cel Dev Biol Plant 39(6):663-668

Kopecky D, Lukaszewski AJ, Gibeault V (2005) Reduction of ploidy level by androgenesis in intergeneric Lolium-Festuca hybrids for turf grass breeding. Crop Sci 45:274-281

Kudirka DT, Schaeffer GW, Baenziger PS (1983) Cytogenetic characteristics of wheat plants regenerated from anther calli of 'Centurk'. Can J Genet Cytol 25:513-517

Lelley T (1992) Triticale, still a promise? Plant Breed 109:1-17

Lelley T (2006) Triticale: a low input cereal with untapped potential. In: Singh RJ, Jauhar PR (eds) Genetic Resources, chromosome engineering, and crop improvement. Taylor and Francis, Roca Baton, pp 395-430

Logue SJ (1996) Genetic stability in microspore-derived doubled haploids. In: Jain SM, Sopory SK, Veilleus RE (eds) In vitro haploid production in higher plants, vol 2. Kluwer, Dordrecht, pp 1-51

Lukaszewski AJ, Gustafson JP (1987) Cytogenetics of triticale. In: Janick J (ed) Plant Breeding Reviews, vol 5. Wiley, New York, pp 41-93

Lukaszewski AJ (1990) Development of aneuploid series in hexaploid triticale. In: Proceedings of 2nd International Triticale Symposium, Passo Fundo, Brazil, CIMMYT, Mexico, D.F., pp. 397-401, 1-5 Oct 1990

Lukaszewski AJ, Xu X (1995) Screening large populations of wheat hybrids by C-banding. Cereal Res Comm 23:9-13

Lukaszewski AJ (1997) Further manipulation by centric misdivision of the 1RS.1BL translocation in wheat. Euphytica 94:257-261

Lukaszewski AJ (2006) Cytogenetically engineered rye chromosomes $1 \mathrm{R}$ to improve bread-making quality of hexaploid triticale. Crop Sci 46:2183-2194

Masoudi-Nejad A, Nasuda S, McIntosh RA, Endo TR (2002) Transfer of rye chromosome segments to wheat by a gametocidal system. Chromosome Res 10:349-357

Metz SG, Sharma TA, Armstrong TA, Mascia PN (1988) Chromosome doubling and aneuploidy in anther-derived plants from two winter wheat lines. Genome 30:177-181 
Nelson MN, Mason AS, Castello MC, Thomson L, Yan G, Cowling WA (2009) Microspore culture preferentially selects unreduced (2n) gametes from an interspecific hybrids of Brassica napus L. $\times$ Brassica cartinata Braun. Theor Appl Genet 119:497-505

Rines HW, Dahleen LS (1990) Haploid oat plants produced by application of maize pollen to emasculated oat florets. Crop Sci 30:1073-1078

Ryöppy PH (1997) Haploidy in triticale. In: Jain SM, Sopory SK, Veilleus RE (eds) In vitro haploid production in higher plants, vol 4. Kluwer, Dordrecht, pp 117-131
Shim YS, Kasha KJ, Simion E, Letarte J (2006) The relationship between induction of embryogenesis and chromosome doubling in microspore cultures. Protoplasma 228:79-86

Struss D, Röbbelen G (1989) Cytological irregularities masked by heterozygosity in triticale. Plant Breed 102:265-272

Zwierzykowski Z, Lukaszewski AJ, Lesniewska A, Naganowska B (1998) Genomic structure of androgenic progeny of pentaploid hybrids, Festuca arundinacea $\times$ Lolium multiflorum. Plant Breed 117:457-462 